\title{
A Polynomial Rooting Approach to Super-Resolution Array Design
}

\author{
Naushad Dowlut, Associate Member, IEEE, and Athanassios Manikas, Member, IEEE
}

\begin{abstract}
This paper is concerned with the design of super-resolution direction finding (DF) arrays that satisfy prespecified performance levels, such as detection-resolution thresholds and Cramér-Rao bounds on error variance. The sensor placement problem is formulated in the framework of subspace-based DF techniques and a novel polynomial rooting approach to the design problem, based on the new concept of the "sensor locator polynomial (SLP)," is proposed. This polynomial is constructed using the prespecified performance levels, and its roots yield the sensor locations of the desired array. The distinguishing feature of the proposed technique is that it hinges on the properties of the array manifold, which plays a central role in all subspace-based DF algorithms.
\end{abstract}

Index Terms-Array manifold, differential geometry, super-resolution array design.

\section{INTRODUCTION}

D URING the past few decades, there has been significant research into source localization algorithms for sensor array signal processing, culminating in the development of subspace-based methods, which asymptotically exhibit infinite resolution, and are hence also referred to as "super-resolution" algorithms. An important issue that has, however, been overlooked is the design of the array structure, which, under practical nonasymptotic conditions, places fundamental limitations on the ultimate performance of the system. The aim of this paper is to investigate and propose a new array design framework tailored to subspace-based DF algorithms.

Array synthesis techniques for other classes of DF algorithms have been investigated in the literature. The common approach is to identify the determining factor in their DF performance. For instance, the design of beamformers is based on the structure of the array gain pattern since the width of the mainlobe governs the resolving power and the height of the sidelobes represent the power leakage from undesired sources. Furthermore, it is known that the beam width is inversely proportional to the array aperture, whereas the sidelobe structure is a function of the number and locations of the sensors [1]. Hence, the design aim for beamformers is to achieve a gain pattern with a narrow mainlobe and low sidelobes. This problem has been extensively

Manuscript received December 28, 1998; revised September 24, 1999. The associate editor coordinating the review of this paper and approving it for publication was Prof. José R. Casar.

N. Dowlut is with the Toronto Design Centre, Motorola Canada, Toronto, Ont., Canada.

A, Manikas is with the Communications and Signal Processing Research Group, Imperial College of Science, Technology and Medicine, London, U.K. (e-mail: a.manikas@ic.ac.uk).

Publisher Item Identifier S 1053-587X(00)04060-5. studied in the literature; see, for instance, [2] and the references therein for a review of the various existing techniques.

The resolving power of the maximum entropy method (MEM), on the other hand, is governed by the number of measurable correlation lags, which is in turn determined by the number and locations of the sensors. An $N$-sensor uniform linear array (ULA), for example, provides $(N-1)$ distinct lags in contrast to the maximum theoretically achievable $N(N-1) / 2$ lags. This discrepancy can be explained in terms of the large number of redundant lags that result from a uniform sensor placement. This consideration has led Lang et al. to propose a design criterion aimed at maximizing the number of different lags while distributing them uniformly within the bounds imposed by the array aperture [3].

Huang et al. presented an approach to array design for the maximum likelihood estimator (MLE) based on minimizing a measure of the DOA estimation errors [4]. Using a Taylor series expansion and retaining the first-order terms only, a linear approximation of the MLE, which is valid for large values of SNR, was obtained, from which an approximate expression for the covariance matrix of the ML estimates was derived. It turned out that the covariance matrix is a function of the true DOA's, the sensor locations, and the noise characteristics. By specifying the number of sensors, the available aperture, the true DOA's, and the noise covariance, it was possible to search for the sensor locations that minimized the determinant of the covariance matrix.

It is important to note that all the above array design techniques involve a search of the sensor locations that satisfy certain algorithm-dependent criteria. Various search techniques have been explored in the literature, for instance, simplex search [2], dynamic programming [5], genetic algorithms [6], and simulated annealing [7], [8]. One common drawback, however, is the computational complexity of the search. In this paper, the design of arrays tailored to subspace-based DF algorithms is addressed. We propose an innovative polynomial rooting approach to the array design problem, which is not only the first super-resolution array design technique, but it also overcomes the computationally intensive search procedure.

The paper is organized as follows. In Section II, the notational conventions used throughout the paper and some preliminaries are presented. In Section III, the array manifold is formally defined, whereas, in Section IV, an analysis of the array manifold using the techniques of the differential geometry of curves is presented, leading to the concept of the sensor locator polynomial introduced and examined in Section V. Both linear and planar array synthesis are investigated. In Section VI, the common performance criteria used in the system specifications 
are examined. The synthesis of fully symmetric and fully asymmetric arrays is then explored in Section VII, and the design of linear and planar arrays based on specified performance levels is presented in Section VIII. Finally, in Section IX, the paper is concluded.

\section{NOTATION AND PRELIMINARIES}

The following notation is used throughout the paper:

$\begin{array}{ll}a & \text { scalar; } \\ \underline{a} & \text { vector; } \\ \mathbf{A} & \text { matrix; } \\ (.)^{T} & \text { transpose; } \\ (.)^{H} & \text { complex conjugate transpose; } \\ \operatorname{fix}_{(.)} & \text {integer part; } \\ \operatorname{ele}{ }_{i j}(\mathbf{A}) & i j \text { th element; } \\ \exp (\underline{a}) & \text { elemental exponential; } \\ \underline{a^{n}} & \text { elemental } n \text {th power; } \\ \operatorname{sum}_{(\underline{a})} & \text { elemental sum; } \\ \|\underline{a}\| & \text { Euclidian norm; } \\ \theta, \phi & \text { azimuth, elevation; } \\ p & \text { generic bearing parameter; } \\ \mathcal{R}^{n} & n \text {-dimensional real space; } \\ \mathcal{C}^{n} & n \text {-dimensional complex space. }\end{array}$

The $(N \times 1)$ complex signal vector $\underline{x}(t) \in \mathcal{C}^{N}$, which is observed at the output of an array of $N$ sensors operating in the presence of $M$ far-field narrowband emitters impinging from bearings $\underline{p}=\left[p_{1}, \cdots, p_{M}\right]^{T}$ and additive noise $\underline{n}(t) \in \mathcal{C}^{N}$, can be modeled as

$$
\underline{x}(t)=\mathbf{A}(\underline{p}) \underline{m}(t)+\underline{n}(t)
$$

where $\underline{m}(t) \in \mathcal{C}^{M}$ is the vector of complex signal envelopes, and $\mathbf{A}(\underline{p}) \in \mathcal{C}^{N \times M}$ is the $(N \times M)$ response matrix defined as

$$
\mathbf{A}(\underline{p}) \triangleq\left[\underline{a}\left(p_{1}\right), \cdots, \underline{a}\left(p_{M}\right)\right]
$$

with $\underline{a}\left(p_{i}\right) \in \mathcal{C}^{N}$, which is known as the "array response vector," denoting the complex array response to a unit amplitude wavefront from direction $p_{i}$. Note that for an azimuth-only DF system, the bearing $p$ represents the azimuth direction $\theta$, and for an azimuth-elevation system, the bearing $p$ represents the azimuth-elevation direction $(\theta, \phi)$.

For an azimuth-only DF system consisting of a linear array of isotropic sensors, which is conventionally taken as lying along the $x$-axis, the response vector is given by

$$
\underline{a}(\theta)=\exp \left(-j \pi \underline{r}_{x} \cos \theta\right)
$$

where $\exp$ (vector) denotes the vector of elemental exponentials, and $\underline{r}_{x} \triangleq\left[r_{1}, r_{2}, \cdots, r_{N}\right]^{T}$ represents the sensor locations in units of half wavelengths. For an azimuth-elevation DF system consisting of a planar array of isotropic sensors, the array response vector is given by

$$
\begin{array}{r}
\underline{a}(\theta, \phi)=\exp \left(-j \pi \underline{r}_{\theta} \cos \phi\right) \\
\underline{r}_{\theta} \triangleq \underline{r}_{x} \cos \theta+\underline{r}_{y} \sin \theta
\end{array}
$$

with $\left[\underline{r}_{x}, \underline{r}_{y}\right]$ denoting the $(x, y)$ coordinates of the sensors in half-wavelength units. Note that $\underline{r}_{\theta}$ represents the projections of the sensors of the planar array onto the line of azimuth $\theta$. Furthermore, the similarity between (3) and (4) leads us to define $\underline{r}_{\theta}$ as the "equivalent linear array (ELA)" of the planar array along the direction $\theta$. This concept will prove invaluable in devising a common method for the design of linear and planar arrays.

\section{ARRAY MAINFOLD}

The array manifold is defined as the locus of all the response vectors over the parameter space $\Omega$

$$
\mathcal{A} \triangleq\{\underline{a}(p): p \in \Omega\}
$$

and it describes a geometrical object in the complex $N$-dimensional space $\mathcal{C}^{N}$. In the case of an azimuth-only DF system, $p=\theta$ and $\Omega=\left[0^{\circ}, 180^{\circ}\right]$ and the single-parameter array manifold traces out a curve in $\mathcal{C}^{N}$, whereas for an azimuth-elevation DF system, $p=(\theta, \phi)$ and $\Omega=\left\{\left[0^{\circ}, 360^{\circ}\right],\left[0^{\circ}, 90^{\circ}\right]\right\}$, and the two-parameter array manifold traces out a surface in $\mathcal{C}^{N}$. An alternative, yet equivalent, $\theta-\phi$ parameterization used in this paper is given by $\Omega=\left\{\left[0^{\circ}, 180^{\circ}\right],\left[0^{\circ}, 180^{\circ}\right]\right.$, and as will be seen, it is extremely useful in that it allows a unified framework for the analysis of the linear and planar array manifolds.

To study the influence of the array manifold on the system's DF performance, the array manifold can be analyzed using the powerful mathematical tools of the differential geometry of curves and surfaces. However, to enable a common analysis of the linear and planar array manifolds, the latter can be treated as a family of curves that make up the surface. A particularly interesting family is the so-called " $\phi$-curves," which are defined as the vector continuum described by the set of all response vectors over the whole elevation space at a particular azimuth $\theta_{\circ}$

$$
C_{\phi, \theta_{\circ}} \triangleq\left\{\underline{a}\left(\theta_{\circ}, \phi\right): \phi \in\left[0^{\circ}, 180^{\circ}\right]\right\} .
$$

The planar array manifold surface can then be alternatively treated as a family of $\phi$-curves spanning the entire azimuth space

$$
\mathcal{A}=\left\{C_{\phi, \theta_{\circ}}: \theta_{\circ} \in\left[0^{\circ}, 180^{\circ}\right]\right\} .
$$

From (4), it is apparent that a $\phi$-line is identical to the manifold of a linear array with sensor locations given by $\underline{r}_{\theta}$, which is, hence, designated as the "equivalent linear array (ELA)."

\section{Differential GeOMEtry of the MAinfold CurVE}

The array manifold is conventionally parameterized in terms of the bearing $p$; however, for the purposes of studying the geometry of the linear array (LA) manifold curve or the planar array manifold $\phi$-curve, parameterization in terms of the arc length, which is the actual physical length in $\mathcal{C}^{N}$, is more suitable. The arc length $s$ is formally defined as

$$
s(p) \triangleq \int_{0}^{p}\left\|\frac{d \underline{a}(p)}{d p}\right\| d p
$$

and the rate of change of arc length is given by

$$
\dot{s}(p) \triangleq \frac{d s}{d p}=\|\underline{\dot{\alpha}}(p)\|
$$


where $p$ represents the azimuth $\theta$ for the LA manifold and the elevation $\phi$ for the $\phi$-curve of the planar array manifold. Using (3) and (4), the following characteristics of the LA manifold and $\phi$-curve can be derived:

$$
\left.\begin{array}{rl}
s_{\mathrm{LA}}(\theta) & =\pi\left\|\underline{r}_{x}\right\|(1-\cos \theta) \\
\dot{s}_{\mathrm{LA}}(\theta) & =\pi\left\|\underline{r}_{x}\right\| \sin \theta \\
s_{\phi}(\phi) & =\pi\left\|\underline{r}_{\theta}\right\|(1-\cos \phi) \\
\dot{s}_{\phi}(\phi) & =\pi\left\|\underline{r}_{\theta}\right\| \sin \phi
\end{array}\right\} \theta \in\left[0^{\circ}, 180^{\circ}\right]
$$

The rate of change of arc length is a local property of the curve, and it has been shown to strongly influence the DF performance of the corresponding array [9]. Another important characteristic of the curve is its total length, which should intuitively have an impact on the ambiguity properties of the array [10] since ambiguities are caused by spurious intersections between the estimated signal subspace and the array manifold. From (11) and (12), the total length of the LA manifold and $\phi$-curve are, respectively, given by

$$
\begin{aligned}
l_{\mathrm{LA}} & =2 \pi\left\|\underline{r}_{x}\right\| \\
l_{\phi} & =2 \pi\left\|\underline{r}_{\theta}\right\| .
\end{aligned}
$$

Furthermore, at every point along the manifold curve, a set of $d$ unit coordinate vectors $\mathbf{U}(s)=\left[\underline{u}_{1}(s), \cdots, \underline{u}_{d}(s)\right] \in \mathcal{C}^{N \times d}$, and $(d-1)$ curvatures $\left\{\kappa_{1}(s), \cdots, \kappa_{d-1}(s)\right\}$ can be defined according to [11]

$$
\mathbf{U}^{\prime}(s)=\mathbf{U}(s) \mathbf{C}(s)
$$

where $(.)^{\prime}$ denotes differentiation with respect to parameter $s$, and $\mathbf{C}(s)$ is the Cartan matrix, which is a real skew-symmetric matrix of the curvatures defined as

$$
\begin{aligned}
\mathbf{C}(s) \triangleq & {\left[\begin{array}{ccccc}
0 & -\kappa_{1}(s) & 0 & \cdots & 0 \\
\kappa_{1}(s) & 0 & -\kappa_{2}(s) & \cdots & 0 \\
0 & \kappa_{2}(s) & 0 & \cdots & 0 \\
\vdots & \vdots & \vdots & \ddots & \vdots \\
0 & 0 & \cdots & \kappa_{d-1}(s) & 0
\end{array}\right] } \\
& \text { with }\left\{\begin{array}{l}
\underline{u}_{1}(s) \triangleq \underline{a}^{\prime}(s) \\
\kappa_{1}(s) \triangleq\left\|\underline{u}_{1}^{\prime}(s)\right\|
\end{array}\right.
\end{aligned}
$$

and $d$ is the dimensionality of the space in which the curve is embedded. It has been shown that in the case of a linear asymmetric array, which is defined as one in which no sensor has a symmetric counterpart with respect to the phase reference (taken at the array centroid), the corresponding manifold occupies the whole of $\mathcal{C}^{N}$, that is, $d=2 N$. If, on the other hand, the linear array is symmetric, that is, all the sensors occur in symmetrical pairs, then $d=N$. Thus, in general, for an $N$-sensor linear array

$$
N \leq d \leq 2 N .
$$

Note that by analogy, the same results pertain to the ELA of a planar array manifold. Equation (14) is a first-order matrix differential equation whose solution can be easily derived as

$$
\mathbf{U}(s)=\mathbf{U}(0) \operatorname{expm}(s \mathbf{C})
$$

where $\mathbf{U}(s)$ is the matrix of coordinate vectors at arc length $s$, and expm(.) denotes the matrix exponential. From (14) and bearing in mind that the coordinate vectors are of unit length, the following expressions for the first three manifold curvatures may be derived:

$$
\begin{aligned}
\kappa_{1} \triangleq\left\|\underline{u}_{1}^{\prime}\right\|=\left\|\underline{\tilde{r}}^{2}\right\| & \\
\kappa_{2} \triangleq\left\|\underline{u}_{2}^{\prime}+\kappa_{1} \underline{u}_{1}\right\| & =\frac{1}{\kappa_{1}}\left\|\underline{\tilde{r}}^{3}-\kappa_{1}^{2} \tilde{\tilde{r}}\right\| \\
\kappa_{3} \triangleq\left\|\underline{u}_{3}^{\prime}+\kappa_{2} \underline{u}_{2}\right\| & =\frac{1}{\kappa_{1} \kappa_{2}}\left\|\underline{\tilde{r}}^{4}-\left(\kappa_{1}^{2}+\kappa_{2}^{2}\right) \underline{\tilde{r}}^{2}\right\| \\
\text { where } \underline{\tilde{r}} & = \begin{cases}\underline{\tilde{r}}_{x}, & \text { linear array } \\
\underline{\tilde{r}}_{\theta}, & \text { planar array. }\end{cases}
\end{aligned}
$$

In general, it can be shown that the $i$ th curvature of an LA manifold or $\phi$-curve is given by the following recursive equation [11]:

$$
\begin{gathered}
\kappa_{i}=\frac{1}{\kappa_{1} \kappa_{2} \cdots \kappa_{i-1}}\left\|\sum_{k=1}^{\operatorname{fix}(i / 2)+1}(-1)^{k-1} b_{i, k} \underline{\underline{r}}^{i-2 k+3}\right\| \\
\text { where }\left\{\begin{array}{l}
\underline{\hat{r}} \triangleq \frac{\underline{r}}{\|\underline{\hat{r}}\|} \text { (normalized sensor locations) } \\
\kappa_{1}=\left\|\underline{\tilde{r}}^{2}\right\| \\
\kappa_{i-1} \neq 0 \\
\operatorname{sum}(\underline{r})=0 \text { (i.e., phase reference }= \\
\quad \text { centroid). }
\end{array}\right.
\end{gathered}
$$

In (21), the coefficients $b_{i, k}$ are given by

$$
\begin{gathered}
b_{i, k}=\sum_{m_{1}=1}^{i-2 k+3} \sum_{m_{2}=m_{1}+2}^{i-2 k+5} \cdots \sum_{m_{k-1}=m_{k-2}+2}^{i-1} \kappa_{m_{1}}^{2} \kappa_{m_{2}}^{2} \cdots \kappa_{m_{k-1}}^{2} \\
\text { with }\left\{\begin{array}{l}
b_{i, 1}=1, \quad i \geq 1 \\
b_{i, 2}=\sum_{m=1}^{i-1} \kappa_{m}^{2}, \quad i>1
\end{array}\right.
\end{gathered}
$$

or, recursively

$$
b_{i, k}=b_{i-1, k}+\kappa_{i-1}^{2} b_{i-2, k-1}, \quad i>2, k>1
$$

with the initial conditions

$$
\left\{\begin{array}{l}
b_{i, 1}=1 \\
b_{2,2}=\kappa_{1}^{2}
\end{array} \quad \forall i \geq 1\right.
$$

Note that the manifold curvatures depend on the relative rather than the absolute sensor spacings and are independent of the arc length parameter. This implies that the manifold curve has the shape of a circular hyperhelix ${ }^{1}$ lying on a complex $N$-dimensional sphere of radius $\sqrt{N}$ in $\mathcal{C}^{N}$.

\footnotetext{
${ }^{1}$ A MATLAB function (hyperlix.m) that calculates the differential geometry parameters of a hyperhelix is available at http://skynet.ee.ic.ac.uk/wwwam/software/arraycommdownload.html.
} 


\section{Sensor Locator Polynominal (SLP)}

Consider the general case of an arbitrary array manifold curve whose $d$ th curvature $\kappa_{d}$ equals zero, and hence, its higher order curvatures are undefined. Note that

$$
d= \begin{cases}2 N-m-1, & \text { if } \exists \text { a sensor at the array centroid } \\
2 N-m, & \text { otherwise }\end{cases}
$$

where $m$ is the number of sensors that occur in symmetrical pairs about the origin or array centroid. For example, in the fully symmetric LA or ELA case, $m=N$, and in the fully asymmetric LA or ELA case, $m=0$. Note that a sensor at the centroid counts as a symmetric sensor, that is, $m=1$; therefore, $d$ is always even, and the zero curvature is always of even order. From (21), it follows that

$$
\left\|\sum_{k=1}^{(d / 2)+1}(-1)^{k-1} b_{d, k} \tilde{r}^{d-2 k+3}\right\|=0 .
$$

The left-hand side of (28) can be reduced to a $(d+1)$ th-order polynomial $p(\tilde{r})$, which is a function of scalar $\tilde{r}$ instead of the vector $\underline{\tilde{r}}$ and is given by

$$
p(\tilde{r})=\tilde{r}^{d+1}-b_{d, 2} \tilde{r}^{d-1}+b_{d, 3} \tilde{r}^{d-3}-\cdots+b_{d,(d / 2)+1} \tilde{r}
$$

where, for example, from (23)

$$
\begin{aligned}
& b_{d, 2}=\sum_{i=1}^{d-1} \kappa_{i}^{2} \\
& b_{d, 3}=\sum_{i=1}^{d-3} \sum_{j=i+2}^{d-1}\left(\kappa_{i} \kappa_{j}\right)^{2}
\end{aligned}
$$

and in general, the coefficients $b_{d, k}$ can be written as

$$
b_{d, k}=\sum_{\substack{m_{1}=1 \\ k>2}}^{d-2 k+3} \sum_{m_{2}=m_{1}+2}^{d-2 k+5} \cdots \sum_{m_{k-1}=m_{k-2}+2}^{d-1} \kappa_{m_{1}}^{2} \kappa_{m_{2}}^{2} \cdots \kappa_{m_{k-1}}^{2}
$$

with $b_{d, 1}=1$ and $b_{d, 2}=\sum_{i=1}^{d-1} \kappa_{i}^{2}$.

Note that the coefficients of the polynomial in (29) are functions of the manifold curvatures, and its roots are the normalized sensor locations $\underline{\tilde{r}}$. Furthermore, $\tilde{r}=0$ is always a root of (29) since a sensor at the centroid does not affect the manifold length and curvatures. In other words, the manifolds of an array with a sensor at the centroid and a similar array, without a sensor at the centroid, are identical except for being embedded in complex spaces of different dimensionalities. However, a sensor at the centroid does affect the ambiguity properties of the array. The polynomial in (29) can, hence, be rewritten as

$$
p(\tilde{r})=\tilde{r}^{d}-b_{d, 2} \tilde{r}^{d-2}+b_{d, 3} \tilde{r}^{d-4}-\cdots+b_{d,(d / 2)+1}
$$

where its coefficients are functions of the manifold curvatures, and its roots are the normalized sensor positions. We call this polynomial the "sensor locator polynomial (SLP)," and since it consists of only the even powers of $\tilde{r}$, its roots always occur in pairs of opposite signs. That is, if $\tilde{r}_{1}$ is a root of the SLP, then so is $-\tilde{r}_{1}$. Then, in the case of fully asymmetric and partially symmetric LA's or ELA's, the roots will actually represent two arrays that are mirror images of each other but whose manifold curvatures are identical. It is obvious in the fully symmetric case that the roots will form a single array since the array and its mirror image are identical. In the fully asymmetric case, the set of roots will consist of two disjoint subsets corresponding to the two mirror arrays. In the partially symmetric case, however, the two subsets will overlap at the symmetric sensors.

A natural question that arises is how can the roots be partitioned into the two mirror arrays. The answer lies in the facts that the synthesized array should be normalized, and the reference is the array centroid, that is, the sum of sensor positions should equal zero. The results obtained so far can be concisely expressed in the following theorem:

Theorem 1-Linear Sensor Locator Polynomial: Given all the $(d-1)$ curvatures $\left\{\kappa_{1}, \cdots, \kappa_{d-1}\right\}$ and the length $l_{m}$ of a manifold curve, the locations of the elements of the LA or ELA (and its mirror image) can be estimated from

$$
\underline{r}_{\text {array } / \text { mirror }}=\frac{l_{m}}{2 \pi} \rho_{\text {array } / \text { mirror }}
$$

where $\rho_{\text {array }}$ and $\rho_{\text {mirror }}$ are two subsets of the set of the roots $\underline{\rho}$ of the following polymomial:

$$
p(\tilde{r})=\tilde{r}^{d}-b_{2} \tilde{r}^{d-2}+b_{3} \tilde{r}^{d-4}-\cdots+b_{(d / 2)+1}
$$

where

$$
\begin{aligned}
& b_{k}=\sum_{m_{1}=1}^{d-2 k+3} \sum_{m_{2}=m_{1}+2}^{d-2 k+5} \cdots \sum_{m_{k-1}=m_{k-2}+2}^{d-1} \kappa_{m_{1}}^{2} \kappa_{m_{2}}^{2} \cdots \kappa_{m_{k-1}}^{2} \\
& k>2
\end{aligned}
$$

with $b_{1}=1, b_{2}=\sum_{i=1}^{d-1} \kappa_{i}^{2}$, and

$$
\left.\begin{array}{l}
\underline{\rho_{\text {array }} \cup \underline{\rho}_{\text {mirror }}=\underline{\rho}} \\
\underline{\rho} \text { array }=-\rho_{\text {mirror }} \\
\left\|\underline{\rho_{\text {array }}}\right\|=\| \underline{\rho}_{\text {mirror }}=1 \\
\operatorname{sum}\left(\underline{\rho}_{\text {array }}\right)=\operatorname{sum}\left(\underline{\rho}_{\text {mirror }}\right)=0
\end{array}\right\} .
$$

The following examples illustrate the theorem.

Example 1-Partially Symmetric LA or ELA: Find the sensor positions of the LA or ELA that has a manifold curve with the following characteristics:

$$
\begin{aligned}
\kappa= & {[0.6727,0.3384,0.4346,0.2335,0.2880} \\
& 0.1791,0.2227] \\
l_{m}= & 19.8692 .
\end{aligned}
$$

From the specified curvatures, the SLP is given by

$p(\tilde{r})=\tilde{r}^{8}-0.9750 \tilde{r}^{6}+0.2494 \tilde{r}^{4}-0.01970 \tilde{r}^{2}+0.0003516$

with roots

$$
\underline{\rho}=[ \pm 0.7905, \pm 0.4746, \pm 0.3610, \pm 0.1582]^{T} .
$$

The subsets of the roots, which satisfy the conditions given by (36) are

$$
\begin{aligned}
\rho_{\text {array }} & =[-0.7905,-0.1582,0.1582,0.3610,0.4746]^{T} \\
\underline{\rho}_{\text {mirror }} & =[-0.4746,-0.3610,-0.1582,0.1582,0.7905]^{T}
\end{aligned}
$$


which give the following LA's and ELA's when scaled to the specified manifold length

$$
\begin{aligned}
\underline{r}_{\text {array }} & =[-2.5,-0.5,0.5,1,1.5]^{T} \\
\underline{r}_{\text {mirror }} & =[-1.5,-1,-0.5,0.5,2.5]^{T} .
\end{aligned}
$$

Note that in this example, the roots of the SLP can be partitioned into two overlapping subsets that constitute a partially symmetric array-mirror array pair.

Although Theorem 1 can synthesize a linear array corresponding to a manifold curve with specified curvatures and length, only the equivalent linear arrays of a planar array are obtainable. The corresponding planar array can, however, be synthesized if two ELA's, say, $\underline{r}_{\theta_{1}}$ and $\underline{r}_{\theta_{2}}$ along two distinct directions, are available, that is, if the curvatures and length of two distinct $\phi$-lines are specified. Fig. 1 illustrates the possible sensor locations of a planar array that can be generated from two given ELA's along the $45^{\circ}$ and $120^{\circ}$ directions, respectively. Recalling that the ELA is the projection of the sensors of the planar array onto a line, then the sensors of the planar array can be positioned at any $N$ of $N^{2}$ grid points, provided that the number of the sensors along any dotted line is equal to the multiplicity of the corresponding projected sensor along that line.

The synthesis of planar arrays from two given ELA's can be mathematically formulated as follows. Consider the case that two normalized ELA's, say, $\underline{\tilde{r}}_{\theta_{1}}$ and $\underline{\tilde{r}}_{\theta_{2}}$, have been identified from the roots of two SLP's constructed from the given curvatures of two $\phi$-lines, say, at $\theta_{1}$ and $\theta_{2}$, respectively. From (5), the two normalized ELA's can be compactly expressed as

$$
\left[\begin{array}{ll}
\underline{\tilde{r}}_{\theta_{1}} & \underline{\tilde{r}}_{\theta_{2}}
\end{array}\right]=\left[\begin{array}{ll}
\tilde{\underline{r}}_{x} & \underline{\tilde{r}}_{y}
\end{array}\right]\left[\begin{array}{ll}
\cos \theta_{1} & \cos \theta_{2} \\
\sin \theta_{1} & \sin \theta_{2}
\end{array}\right]
$$

from which the normalized sensor positions of the planar array are simply given by

$$
\begin{aligned}
{\left[\begin{array}{ll}
\underline{\tilde{r}}_{x} & \tilde{\underline{r}}_{y}
\end{array}\right]=} & \frac{1}{\sin \left(\theta_{2}-\theta_{1}\right)}\left[\begin{array}{ll}
\underline{\tilde{r}}_{\theta_{1}} & \underline{\tilde{r}}_{\theta_{2}}
\end{array}\right] \\
& \cdot\left[\begin{array}{rr}
\sin \theta_{2} & -\cos \theta_{2} \\
-\sin \theta_{1} & \cos \theta_{1}
\end{array}\right] \text { where } \\
& \quad\left(\theta_{2}-\theta_{1}\right) \neq n 180^{\circ}, n=0, \pm 1, \pm 2, \cdots .
\end{aligned}
$$

The actual sensor locations of the planar array are obtained by "denormalizing" $\underline{\widetilde{r}}_{\theta_{1}}$ and $\underline{\tilde{r}}_{\theta_{2}}$ in the previous expression as follows:

$$
\begin{aligned}
& {\left[\begin{array}{ll}
\underline{r}_{x} & \underline{r}_{y}
\end{array}\right]=\frac{1}{\sin \left(\theta_{2}-\theta_{1}\right)}\left[\frac{l_{\phi}\left(\theta_{1}\right)}{2 \pi} \underline{\tilde{r}}_{\theta_{1}} \frac{l_{\phi}\left(\theta_{2}\right)}{2 \pi} \underline{\tilde{r}}_{\theta_{2}}\right]} \\
& \text { - }\left[\begin{array}{rr}
\sin \theta_{2} & -\cos \theta_{2} \\
-\sin \theta_{1} & \cos \theta_{1}
\end{array}\right] \quad \text { where } \\
& \left(\theta_{2}-\theta_{1}\right) \neq n 180^{\circ}, n=0, \pm 1, \pm 2, \cdots \text {. }
\end{aligned}
$$

It can be seen that (39) generates only a single planar array from the two ELA's, whereas it is obvious from Fig. 1 that the solution cannot be unique. The key to this apparent contradiction lies in the subtle observation that the different planar arrays give rise to different permutations of the elements of the ELA's, that is, the order of the elements of the ELA's matters. The re-

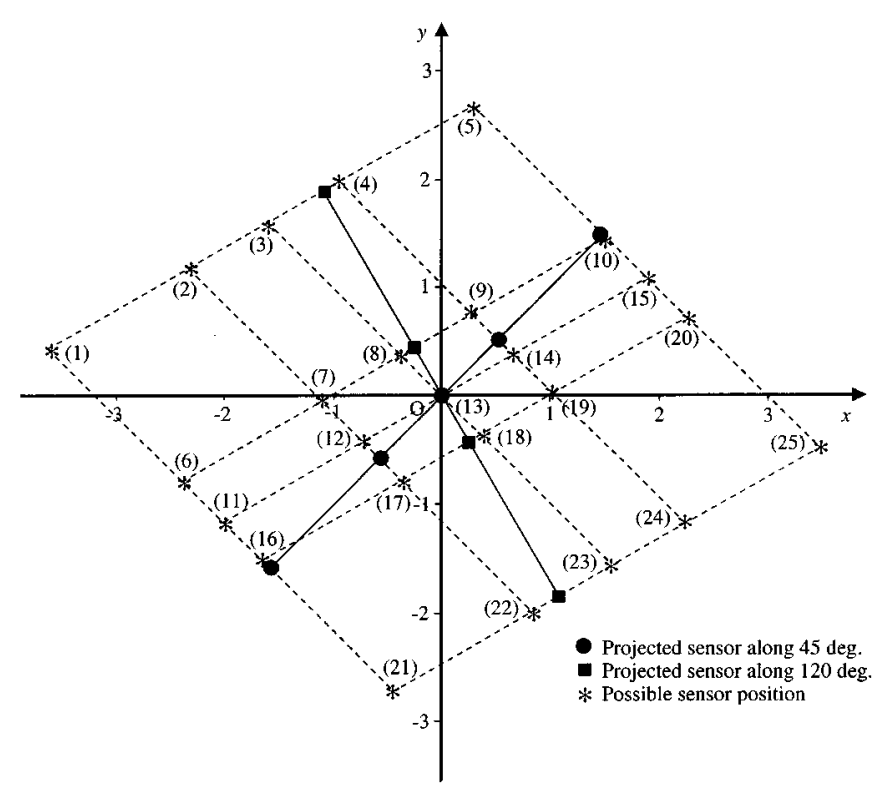

Fig. 1. ELA's and possible sensor locations.

sults pertaining to the planar array case are summarized in the following theorem.

Theorem 2-Planar Sensor Locator Polynomial: Given the lengths $l_{\phi}\left(\theta_{1}\right)$ and $l_{\phi}\left(\theta_{2}\right)$ and all the $(d-1)$ curvatures $\kappa_{\phi}\left(\theta_{1}\right)$ and $\kappa_{\phi}\left(\theta_{2}\right)$ of two distinct $\phi$-curves of a planar array manifold, the sensor locations of a possible planar array can be obtained using the expression

$$
\begin{aligned}
{\left[\begin{array}{ll}
\underline{r}_{x} & \underline{r}_{y}
\end{array}\right]=} & \frac{1}{\sin \left(\theta_{2}-\theta_{1}\right)}\left[\frac{l_{\phi}\left(\theta_{1}\right)}{2 \pi} \underline{\rho}_{\theta_{1}} \frac{l_{\phi}\left(\theta_{2}\right)}{2 \pi} \underline{\rho}_{\theta_{2}}\right] \\
& \cdot\left[\begin{array}{rr}
\sin \theta_{2} & -\cos \theta_{2} \\
-\sin \theta_{1} & \cos \theta_{1}
\end{array}\right]
\end{aligned}
$$

where $\left(\theta_{2}-\theta_{1}\right) \neq n 180^{\circ}, n=0, \pm 1, \pm 2, \cdots$ where $\rho_{\theta_{i}}$ represents a permutation of either of the subsets $\rho_{\text {array }}$ or $\rho_{\text {mirror }}$, which is given by the roots of the following polynomial:

$$
p\left(\tilde{r}_{\theta}\right)=\tilde{r}_{\theta}^{d}-b_{2}(\theta) \tilde{r}_{\theta}^{d-2}+b_{3}(\theta) \tilde{r}_{\theta}^{d-4}-\cdots+b_{(d / 2)+1}(\theta)
$$

where

$$
\begin{aligned}
& b_{k}(\theta)= \sum_{m_{1}=1}^{d-2 k+3} \sum_{m_{2}=m_{1}+2}^{d-2 k+5} \cdots \sum_{m_{k-1}=m_{k-2}+2}^{d-1} \cdots>2 \\
& \cdot \kappa_{m_{1} \phi}^{2} \kappa_{m_{2} \phi}^{2} \cdots \kappa_{m_{k-1} \phi}^{2}, \quad k>2
\end{aligned}
$$

and $b_{1}(\theta)=1, b_{2}(\theta)=\sum_{i=1}^{d-1} \kappa_{i \phi}^{2}$, with $\rho_{\text {array }}$ and $\rho_{\text {mirror }}$ satisfying the conditions of (36).

The choice of a particular configuration is then governed by further considerations such as the size and shape of the available site and the ambiguity properties of the candidate configurations. In the next section, the determination of the manifold characteristics from the performance specifications is addressed. This will then enable the synthesis of arrays that satisfy prespecified performance levels.

\section{Performance CRITERIA}

Performance criteria constitute an essential ingredient of the design process. In the case of super-resolution DF array de- 
sign, popular performance criteria include the detection, resolution, and estimation performance inherent in the sensor configuration. The detection performance refers to the ability of the DF system to correctly enumerate the emitters present in the environment; this information is then used to partition the observation space into the signal and noise subspaces, which are subsequently employed in the estimation process. The resolving power of the array is assessed by its ability to distinguish between closely spaced sources. For a spectrum-based DF algorithm such as MUSIC, two sources are said to be resolved if the spectrum exhibits two distinct peaks at or near the two actual emitter directions. The estimation performance, on the other hand, refers to the error incurred when estimating the DOA's of the emitters. This is usually assessed using the well-known Cramér-Rao bound (CRB), which represents the minimum achievable estimation error variance of any unbiased estimator.

Recall that when the exact covariance matrix is available, the signal and noise subspaces can be accurately determined, arbitrarily close emitters can be successfully detected and resolved, and their DOA's can be perfectly estimated. In practice, however, when only a finite amount of noisy data is available, it has been shown that the detection, resolution, and estimation performance depend not only on the observation interval and SNR but also on the physical characteristics of the array via the local structure of the array manifold [9], [12], [13]. For instance, the SNR thresholds of detection and resolution of two equipower emitters, over an observation interval of $L$ snapshots, are, respectively, given by [12]

$$
\begin{aligned}
\mathrm{SNR}_{\mathrm{DET}} & =\frac{2}{L \Delta s^{2}} \\
\mathrm{SNR}_{\mathrm{RES}} & =\frac{32}{L \Delta s^{4}\left(\hat{\kappa}_{1}^{2}-\frac{1}{N}\right)}
\end{aligned}
$$

where $\Delta s$ is the arc length separation of the two emitters, which, from (11) or (12), can be written as

$$
\Delta s=\pi|| \underline{r}||\left|\cos p_{2}-\cos p_{1}\right|
$$

and $1 / \hat{\kappa}_{1}$ is the radius of the circular arc approximation of the manifold given by

$$
\hat{\kappa}_{1}=\sqrt{\kappa_{1}^{2}-\left[\operatorname{sum}\left(\underline{\tilde{r}}^{3}\right)\right]^{2}} .
$$

Note that the curvature of the circular arc approximation is identical to the manifold's first curvature if the LA or ELA is symmetric.

Furthermore, the one- and two-source CRB's on the DOA estimation error variance of any unbiased estimator have been shown to be given by [13]

$$
\begin{aligned}
\mathrm{CRB}_{1}\left(p_{1}\right) & =\frac{1}{2 L \times \mathrm{SNR}_{1} \dot{s}\left(p_{1}\right)^{2}} \\
\mathrm{CRB}_{2}\left(p_{1}\right) & =\frac{2}{L \times \mathrm{SNR}_{1} \dot{s}\left(p_{1}\right)^{2} \Delta s^{2}\left(\hat{\kappa}_{1}^{2}-\frac{1}{N}\right)}
\end{aligned}
$$

where $\dot{s}\left(p_{1}\right)$ is the rate of change of arc length given by (11) or (12).
From (43) and (46), if the desired detection, resolution, or DOA estimation performance is specified, then the manifold length and first curvature of the corresponding symmetric LA or ELA can be calculated as

$$
\begin{aligned}
& l_{m}=\left\{\begin{array}{l}
\frac{1}{\sin p \sin \frac{\Delta p}{2}} \sqrt{\frac{2}{L \times \mathrm{SNR}_{\mathrm{DET}}}} \\
\sqrt{\frac{2}{L \times \mathrm{SNR} \sin ^{2} p_{1} \mathrm{CRB}_{1}\left(p_{1}\right)}}
\end{array}\right. \\
& \kappa_{1}=\left\{\begin{array}{l}
\sqrt{\frac{32}{L \times \mathrm{SNR}_{\mathrm{RES}}\left(l_{m} \sin p \sin \frac{\Delta p}{2}\right)^{4}}+\frac{1}{N}} \\
\sqrt{\frac{2}{L \times \mathrm{SNR}_{1} \dot{s}\left(p_{1}\right)^{2} \Delta s^{2} \mathrm{CRB}_{2}\left(p_{1}\right)}+\frac{1}{N}}
\end{array}\right. \\
& \text { where }\left\{\begin{array}{l}
\Delta s=l_{m} \sin \left(p_{1}+\frac{\Delta p}{2}\right) \sin \frac{p_{1}}{2} \\
\dot{s}\left(p_{1}\right)=\frac{l_{m}}{2} \sin p_{1} .
\end{array}\right.
\end{aligned}
$$

It is important to note that the standard performance criteria considered only yield the first manifold curvature $\kappa_{1}$. Recalling that Theorems 1 and 2 require the whole set of curvatures to synthesize the corresponding LA or ELA, new techniques then have to be devised to design arrays based on the first curvature only. In the next section, it is shown that for the special cases of fully symmetric and fully asymmetric LA's or ELA's, it is possible to construct the SLP using a small subset of all the manifold curvatures.

$$
\begin{aligned}
& \text { VII. FULLY SYMMETRIC AND FULLY ASYMMETRIC LA'S OR } \\
& \text { ELA'S } \\
& \text { Consider the sensor locator polynomial given by } \\
& p(\tilde{r})=\tilde{r}^{d}+c_{1} \tilde{r}^{d-2}+c_{2} \tilde{r}^{d-4}+\cdots+c_{d / 2} \\
& \text { where } c_{m} \triangleq(-1)^{m} b_{d, m+1} \\
& =f\left\{\kappa_{1}, \kappa_{2}, \cdots, \kappa_{d-1}\right\}
\end{aligned}
$$

and $f\{$.$\} is interpreted as "is a function of," that is, the coef-$ ficients of the SLP are functions of all the $(d-1)$ manifold curvatures. The problem is to evaluate the coefficients when not all of the curvatures are known.

It is shown in Appendix A that the $m$ th coefficient of the SLP can be written as

$$
\begin{gathered}
c_{m}=-\frac{1}{m} \sum_{i=0}^{m-1} c_{i} \xi_{2(m-i)} \quad \text { where } c_{0} \triangleq 1 \\
\xi_{n} \triangleq \sum_{j=1}^{d / 2} \rho_{j}^{n}=\frac{1}{2} \operatorname{sum}\left(\underline{\rho}^{n}\right) .
\end{gathered}
$$

Note that $\xi_{n}$ represents the sum of the $n$th power of all the positive or negative roots (since $n$ is always even) of the SLP, and

$$
c_{m}=f\left\{\xi_{2}, \xi_{4}, \cdots, \xi_{2 m}\right\} .
$$

Interestingly, the sums of similar powers of the normalized sensor locations $\left[\operatorname{sum}\left(\underline{\hat{r}}^{n}\right)\right]$ can be expressed in terms of the 
manifold curvatures, as can be realized by expanding the explicit expressions in (19)

$$
\begin{aligned}
& \operatorname{sum}\left(\underline{\tilde{r}}^{4}\right)=\kappa_{1}^{2} \\
& \operatorname{sum}\left(\underline{\tilde{r}}^{6}\right)=\kappa_{1}^{2}\left(\kappa_{1}^{2}+\kappa_{2}^{2}\right) \\
& \operatorname{sum}\left(\underline{\tilde{r}}^{8}\right)=\left(\kappa_{1} \kappa_{2} \kappa_{3}\right)^{2}+\kappa_{1}^{2}\left(\kappa_{1}^{2}+\kappa_{2}^{2}\right)^{2}
\end{aligned}
$$

and, as proved in Appendix B, in general, the sum of the $n$th power of the normalized sensor locations, where $n$ is even, can be expressed in terms of the sums of lower even powers of the normalized sensor locations and the first $((n / 2)-1)$ curvatures as follows:

$$
\begin{aligned}
\operatorname{sum}\left(\underline{\hat{r}}^{n}\right)= & \left(\kappa_{1} \kappa_{2} \cdots \kappa_{(n / 2)-1}\right)^{2}+2 b_{1} b_{2} \operatorname{sum}\left(\underline{\tilde{r}}^{n-2}\right) \\
& -\left(2 b_{1} b_{3}+b_{2}^{2}\right) \operatorname{sum}\left(\underline{\hat{r}}^{n-4}\right)+\cdots+\eta_{p} \operatorname{sum}\left(\underline{\hat{r}}^{n-p}\right) \\
& +\cdots-b_{\text {fix }(((n / 2)-1) / 2)+1}^{2} \underline{\hat{r}}_{x i}^{q}
\end{aligned}
$$

where $b_{k}, q$, and $\eta_{p}$ are defined in Appendix B.

It is worth noting that

$$
\operatorname{sum}\left(\underline{\tilde{r}}^{n}\right)=f\left\{\kappa_{1}, \kappa_{2}, \cdots, \kappa_{(n / 2)-1}\right\} .
$$

The next step is to find out the relationship between $\xi_{n}$ and $\operatorname{sum}\left(\tilde{r}^{n}\right)$. As previously mentioned, the roots of the SLP represent a normalized array together with its mirror image about the centroid, and hence, it can be deduced that for the fully symmetric and fully asymmetric arrays

$$
\begin{aligned}
\xi_{n}^{\text {sym }} & =\frac{1}{2} \operatorname{sum}\left(\underline{\tilde{r}}^{n}\right) \\
\xi_{n}^{\text {asym }} & =\operatorname{sum}\left(\underline{\tilde{r}}^{n}\right)
\end{aligned}
$$

and therefore, from (51) and (54)

$$
c_{m}=f\left\{\kappa_{1}, \kappa_{2}, \cdots, \kappa_{m-1}\right\}
$$

that is, the $m$ th coefficient of the SLP of a fully symmetric or fully asymmetric array is a function of only the first $(m-1)$ manifold curvatures. In particular, $c_{(d / 2)}=f\left\{\kappa_{1}, \kappa_{2}, \cdots, \kappa_{(d / 2)-1}\right\}$, and based on the previous discussion, the following theorem is evident.

Theorem 3-Fully Symmetric and Fully Asymmetric SLP: The sensor locator polynomial of a fully symmetric or fully asymmetric array can be formed using only the first $((d / 2)-1)$ of the $(d-1)$ manifold curvatures.

This result is of great significance since it allows the construction of the SLP using only a subset of the curvatures. Recalling that

$$
\begin{aligned}
d_{\text {sym }} & = \begin{cases}N-1, & \text { if } \exists \text { a sensor at array centroid } \\
N, & \text { otherwise }\end{cases} \\
d_{\text {asym }} & = \begin{cases}2 N-1, & \text { if } \exists \text { a sensor at array centroid } \\
2 N, & \text { otherwise }\end{cases}
\end{aligned}
$$

two corollaries follow immediately.

Corollary 3.1: Given $N$ isotropic sensors, the number of curvatures required to design $\mathrm{a}^{2}$

1) fully symmetric array $= \begin{cases}\frac{N-3}{2}, & N \text { odd } \\ \frac{N}{2}-1, & N \text { even }\end{cases}$

2) fully asymmetric array $=N-1$.

${ }^{2} N$ odd implies that one sensor is fixed at the array centroid, so that the number of sensors to be positioned is effectively $N-1$
Corollary 3.2: If $n$ curvatures $\left\{\kappa_{1}, \kappa_{2}, \cdots, \kappa_{n}\right\}$ are known, then the minimum number of sensors required to design an array with the given manifold curvatures is $(n+1)$, and the array has to be fully asymmetric. If a fully symmetric normalized array is desired, then the number of sensors required is $2(n+1)$ if no sensor is placed at the centroid.

Note that for the same number of sensors, the fully asymmetric array synthesis requires about twice the number of curvatures than the fully symmetric array design. This is to be expected since the fully symmetric array design procedure amounts to the placement of only half the number of sensors.

In the partially symmetric case, however, there exists no such relationship as (55) between $\xi_{n}^{\mathrm{psym}}$ and $\operatorname{sum}\left(\underline{\underline{r}}^{n}\right)$ because of the overlap of the roots, and therefore, (50) is not applicable. Considering the specific example of $\xi_{2}$, which is defined as

$$
\xi_{2} \triangleq \sum_{i=1}^{d / 2} \rho_{i}^{2}=\frac{1}{2}\|\underline{\rho}\|^{2}
$$

it can be easily verified that

$$
\xi_{2}^{\mathrm{sym}}=\frac{1}{2} ; \quad \xi_{2}^{\mathrm{asym}}=1 ; \quad \frac{1}{2}<\xi_{2}^{\mathrm{psym}}<1 .
$$

Note that the exact value of $\xi_{2}^{\mathrm{psym}}$ cannot be determined.

Using (50), (52), and (55), the first three coefficients of the SLP for a fully symmetric array are given by

$$
\begin{aligned}
c_{1}^{\mathrm{sym}} & =-c_{o} \xi_{2}^{\mathrm{sym}}=-\frac{1}{2} \\
c_{2}^{\mathrm{sym}} & =-\frac{1}{2}\left(c_{o} \xi_{4}^{\mathrm{sym}}+c_{1} \xi_{2}^{\mathrm{sym}}\right)=\frac{1}{8}\left(1-2 \kappa_{1}^{2}\right) \\
c_{3}^{\mathrm{sym}} & =-\frac{1}{3}\left(c_{o} \xi_{6}^{\mathrm{sym}}+c_{1} \xi_{4}^{\mathrm{sym}}+c_{2} \xi_{2}^{\mathrm{sym}}\right) \\
& =-\frac{1}{48}\left(8 \kappa_{1}^{2}\left(\kappa_{1}^{2}+\kappa_{2}^{2}\right)-6 \kappa_{1}^{2}+1\right)
\end{aligned}
$$

and for a fully asymmetric array

$$
\begin{aligned}
c_{1}^{\text {asym }} & =-c_{o} \xi_{2}^{\text {asym }}=-1 \\
c_{2}^{\text {asym }} & =-\frac{1}{2}\left(c_{o} \xi_{4}^{\text {asym }}+c_{1} \xi_{2}^{\text {asym }}\right)=\frac{1}{2}\left(1-\kappa_{1}^{2}\right) \\
c_{3}^{\text {asym }} & =-\frac{1}{3}\left(c_{o} \xi_{6}^{\text {asym }}+c_{1} \xi_{4}^{\text {asym }}+c_{2} \xi_{2}^{\text {asym }}\right) \\
& =-\frac{1}{6}\left(2 \kappa_{1}^{2}\left(\kappa_{1}^{2}+\kappa_{2}^{2}\right)-3 \kappa_{1}^{2}+1\right) .
\end{aligned}
$$

\section{ARRAY DESIGN-BASED ON $\kappa_{1}$ ONLY}

The core of the proposed array design approach is the "sensor locator polynomial" (SLP), which is a polynomial constructed using the curvatures of the manifold and whose roots yield the desired sensor locations. The construction and properties of this polynomial has been investigated in detail in Section V. In this section, a special case of the proposed general sensor locator polynomial theory, based on only the first curvature and the length of the manifold, will be examined. Under such circumstances, the following special case result of the general sensor locator polynomial theory applies.

If the length $l_{m}$ and only the first curvature $\kappa_{1}$ of a hyperhelical manifold is known, then it is possible to synthesize a corresponding five-sensor symmetric LA or ELA given by

$$
\underline{r}=\frac{l_{m}}{2 \pi} \rho
$$

where $\rho$ is the set of roots of the following sensor locator polynomial:

$$
\begin{aligned}
& p(\tilde{r})=\tilde{r}^{\tilde{s}}+c_{1} \tilde{r}^{3}+c_{2} \tilde{r} \\
& \quad \text { with } c_{1}=-0.5 ; c_{2}=\frac{1}{8}\left(1-2 \kappa_{1}^{2}\right) .
\end{aligned}
$$




\section{A. Linear Array Design Example}

Consider the following specifications for the design of a fivesensor linear array.

Specifications for Design Example 1: Design a five-sensor linear array, which, for an observation interval of 100 snapshots, exhibits a one-source $\mathrm{CRB}$ of $0.15^{\circ}$ on the standard deviation of the DOA estimate of an emitter at $45^{\circ}$ with SNR $=10(10 \mathrm{~dB})$, and a two-source $\mathrm{CRB}$ of $1.5^{\circ}$ on the standard deviation of the DOA estimate of the same emitter in the presence of a second uncorrelated equipower emitter at $49^{\circ}$.

Note that under an identical scenario, a standard five-sensor uniform linear array (ULA) exhibits a one-source CRB of $0.1824^{\circ}$ and a two-source $\mathrm{CRB}$ of $1.9183^{\circ}$, according to the well known expressions reported in [14].

The manifold length and first curvature of the desired array can be calculated by applying (47) and (48) to the design specifications and are given by $l_{m}=24.1580, \kappa_{1}=0.5525$. From (63), the corresponding SLP can then be written as

$$
p(\tilde{r})=\tilde{r}^{\tilde{s}}-0.5 \tilde{r}^{3}+0.0487 \tilde{r}
$$

with roots $\underline{\rho}=[0, \pm 0.3640, \pm 0.6062]^{T}$, which actually constitutes the normalized version of the desired array. After scaling using (62), the desired array in half-wavelength units is given by

$$
\underline{r}_{x}=[-2.3308,-1.3997,0,1.3997,2.3308]^{T} .
$$

As a check of the validity of the design procedure, the CRB's of the proposed array are computed using the exact expression given in [14] and are $\sqrt{\mathrm{CRB}_{1}}=0.1508^{\circ}$ and $\sqrt{\mathrm{CRB}_{2}}=$ $1.4836^{\circ}$ and are found to match the specifications very closely.

\section{B. Planar Array Design Example}

Consider the following set of specifications for the design of a five-sensor planar array.

Specifications for Design Example 2: Design a five-sensor planar array, which for an observation interval of 100 snapshots and two uncorrelated equipower emitters with SNR $=10$ (10 $\mathrm{dB}$ ) about an elevation $\phi$ and common azimuth $\theta_{0}$, exhibits the following ultimate detection and resolution thresholds.

Note that the detection and resolution thresholds are expressed in terms of the minimum allowable separation of two emitters for successful detection and resolution, respectively. From the detection and resolution threshold specifications in Table I, the lengths and first curvatures of the $\phi$-curves at the given azimuths $\theta_{\circ}$ can be calculated using (47) and (48) as follows:

$$
\begin{aligned}
l_{\phi}\left(45^{\circ}\right) & =20.4988, \quad \kappa_{1 \phi}\left(45^{\circ}\right)=0.6393 \\
l_{\phi}\left(120^{\circ}\right) & =19.7250, \quad \kappa_{1 \phi}\left(120^{\circ}\right)=0.6733 .
\end{aligned}
$$

Using (63), the corresponding SLP's and ELA's) are, respectively, given by

$$
\begin{aligned}
& p(\tilde{r})=\tilde{r}^{\tilde{s}}-0.5 \tilde{r}^{3}+0.02282 \tilde{r} \\
& p(\tilde{r})=\tilde{r}^{\tilde{s}}-0.5 \tilde{r}^{3}+0.01167 \tilde{r}
\end{aligned}
$$

and

$$
\begin{aligned}
\underline{r}_{45^{\circ}} & =[0, \pm 0.7353, \pm 2.1866]^{T} \\
\underline{r}_{120^{\circ}} & =[0, \pm 0.4916, \pm 2.1647]^{T}
\end{aligned}
$$

TABLE I

DESIRED DETECTION AND RESOLUTION THRESHOLDS

\begin{tabular}{c|c|c|c}
\hline \multicolumn{4}{c}{$L=100, \mathrm{SNR}=10 \mathrm{~dB}$} \\
\hline$\theta_{\circ}$ & $\phi$ & $(\Delta \phi)_{\text {DET }}$ & $(\Delta \phi)_{\text {RES }}$ \\
\hline $45^{\circ}$ & $30^{\circ}$ & $0.5^{\circ}$ & $7^{\circ}$ \\
\hline $120^{\circ}$ & $60^{\circ}$ & $0.3^{\circ}$ & $4^{\circ}$ \\
\hline
\end{tabular}

TABLE II

All Possible Sensor Locations

\begin{tabular}{c|c|c||c|c|c}
\hline Sensor & $r_{x}$ & $r_{y}$ & Sensor & $r_{x}$ & $r_{y}$ \\
\hline 1 & -3.5451 & 0.4528 & 14 & 0.6593 & 0.3806 \\
\hline 2 & -2.2439 & 1.2041 & 15 & 1.9605 & 1.1319 \\
\hline 3 & -1.5847 & 1.5847 & 16 & -1.6006 & -1.4917 \\
\hline 4 & -0.9254 & 1.9653 & 17 & -0.2994 & -0.7405 \\
\hline 5 & 0.3758 & 2.7165 & 18 & 0.3599 & -0.3599 \\
\hline 6 & -2.3203 & -0.7720 & 19 & 1.0191 & 0.0207 \\
\hline 7 & -1.0191 & -0.0207 & 20 & 2.3203 & 0.7720 \\
\hline 8 & -0.3599 & 0.3599 & 21 & -0.3758 & -2.7165 \\
\hline 9 & 0.2994 & 0.7405 & 22 & 0.9254 & -1.9653 \\
\hline 10 & 1.6006 & 1.4917 & 23 & 1.5847 & -1.5847 \\
\hline 11 & -1.9605 & -1.1319 & 24 & 2.2439 & -1.2041 \\
\hline 12 & -0.6593 & -0.3806 & 25 & 3.5451 & -0.4528 \\
\hline 13 & 0 & 0 & & & \\
\hline
\end{tabular}

The sensor locations of the desired planar array can now be calculated from the ELA's using (39):

$$
\begin{aligned}
{\left[\begin{array}{ll}
\underline{r}_{x} & \underline{r}_{y}
\end{array}\right]=} & \frac{1}{\sin \left(120^{\circ}-45^{\circ}\right)}\left[\begin{array}{lr}
\underline{r}_{45^{\circ}} & \underline{r}_{120^{\circ}}
\end{array}\right] \\
& \cdot\left[\begin{array}{cc}
\sin \left(120^{\circ}\right) & -\cos \left(120^{\circ}\right) \\
-\sin \left(45^{\circ}\right) & \cos \left(45^{\circ}\right)
\end{array}\right] .
\end{aligned}
$$

Recall that all the permutations of the elements of $\underline{r}_{45^{\circ}}$ and $\underline{r}_{120^{\circ}}$ should be taken into account in order to generate the set of planar arrays satisfying the specified manifold parameters. Alternatively, the possible sensor locations can be graphically determined as illustrated in Fig. 1, where the $N$ sensors can be positioned at any of $N^{2}$ grid points indicated by the asterisks, making sure, however, that the number of sensors along any dotted line equals the multiplicity of the corresponding projected sensor. The possible sensor locations of the desired array for the design example under consideration are tabulated in Table II.

Assuming that all the projected sensors have multiplicity equal to one, it can be shown that the total number of possible $N$-sensor arrangements is equal to $N$ !. Furthermore, if the ELA's are symmetric, as is the present case, then both an array and its $180^{\circ}$ rotated counterpart can be constructed. Fig. 2 illustrates such a pair from the current example. From a performance point of view, the two arrays are equivalent, and hence, the total number of possible designs is effectively equal to $N ! / 2$. In the current design example, this means that the solution set will consist of 60 possible arrays. 

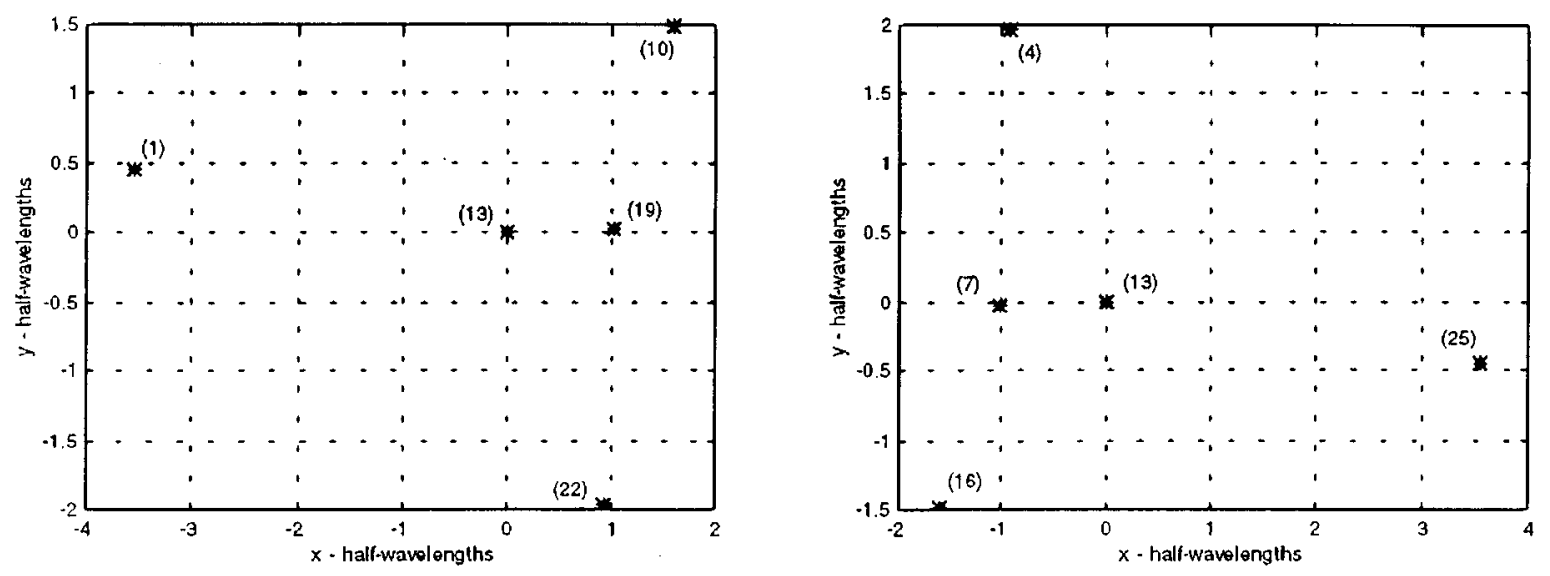

Fig. 2. Two equivalent candidate designs of Design Example 3.

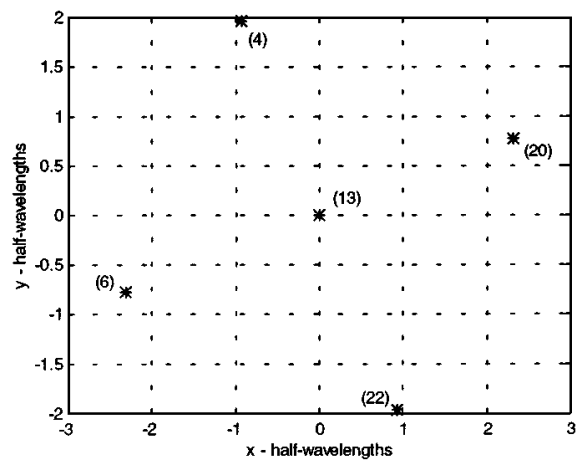

Fig. 3. Possible planar array design.

Fig. 3 illustrates one of the possible designs that can be shown using (43) to match the specifications in Table I exactly.

\section{CONCLUSION}

In this paper, an array design approach tailored to subspacebased DF algorithms was proposed. The core of the design approach is the sensor locator polynomial (SLP), which is constructed using the manifold curvatures and whose roots yield the normalized sensor locations of the desired array. It was shown that design specifications in terms of common performance criteria such as detection, resolution, and Cramér-Rao bounds can yield the manifold length and first curvature, and a procedure to design fully symmetric and fully asymmetric arrays with a small subset of the curvatures was presented. Finally, the concepts and techniques presented in the paper were illustrated by a linear and a planar array design example.

\section{APPENDIX A}

DERIVATION OF SLP COEFFICIENTS IN TERMS OF SUMS OF POWERS OF ROOTS

Consider the sensor locator polynomial given by

$$
\begin{aligned}
& p(\tilde{r})=\tilde{r}^{d}+c_{1} \tilde{r}^{d-2}+c_{2} \tilde{r}^{d-4}+\cdots+c_{d / 2} \\
& \text { where } c_{m} \triangleq(-1)^{m} b_{d, m+1} \\
&=f\left\{\kappa_{1}, \kappa_{2}, \cdots, \kappa_{d-1}\right\}
\end{aligned}
$$

and $f\{$.$\} is interpreted as "is a function of." The problem is$ to evaluate the coefficients when not all of the curvatures are known. A useful theorem by Newton [15] states the following.

Theorem: The sums of the similar powers of the roots of an equation can be expressed rationally in terms of the coefficients.

To apply Newton's theorem to the SLP, the following lemma, which is proved in [15], will be required.

Lemma: The first derivative of an $n$th degree polynomial

$$
P(x) \equiv p_{\circ} x^{n}+p_{1} x^{n-1}+p_{2} x^{n-2}+\cdots+p_{n-1} x+p_{n}
$$

with roots $\underline{\rho}=\left[\rho_{1}, \rho_{2}, \cdots, \rho_{n}\right]^{T}$ can be written as

$$
P^{\prime}(x)=\frac{P(x)}{x-\rho_{1}}+\frac{P(x)}{x-\rho_{2}}+\cdots+\frac{P(x)}{x-\rho_{n}} .
$$

Applying the lemma to the SLP given in (67) results in the following expression for the first derivative of the SLP with respect to $\tilde{r}$ :

$$
p^{\prime}(\tilde{r})=\frac{p(\tilde{r})}{\tilde{r}-\rho_{1}}+\frac{p(\tilde{r})}{\tilde{r}-\rho_{2}}+\cdots+\frac{p(\tilde{r})}{\tilde{r}-\rho_{d}} .
$$

Recalling that the roots of the SLP occur in pairs of opposite signs, (68) can be reduced to

$$
\frac{p^{\prime}(\tilde{r})}{2 \tilde{r}}=\frac{p(\tilde{r})}{\tilde{r}^{2}-\rho_{1}^{2}}+\frac{p(\tilde{r})}{\tilde{r}^{2}-\rho_{2}^{2}}+\cdots+\frac{p(\tilde{r})}{\tilde{r}^{2}-\rho_{d / 2}^{2}} .
$$

Noting that $\left(\tilde{r}^{2}-\rho_{i}^{2}\right)$ is a factor of $p(\tilde{r})$, a generic term of (69) can be expressed as

$$
\frac{p(\tilde{r})}{\tilde{r}^{2}-\rho_{i}^{2}} \equiv q_{\circ} \tilde{r}^{d-2}+q_{1} \tilde{r}^{d-4}+\cdots+q_{(d / 2)-2} \tilde{r}^{2}+q_{(d / 2)-1}
$$

where the coefficients $q_{\circ}, q_{1}, \cdots, q_{(d / 2)-1}$ can be determined by multiplying out (70) as follows:

$$
\begin{aligned}
p(\tilde{r})= & q_{\circ} \tilde{r}^{d}+\left(q_{1}-q_{\circ} \rho_{i}^{2}\right) \tilde{r}^{d-4}+\left(q_{2}-q_{1} \rho_{i}^{2}\right) \tilde{r}^{d-6} \\
& +\cdots+\left(q_{(d / 2)-1}-q_{(d / 2)-2} \rho_{i}^{2}\right) \tilde{r}^{2}-q_{(d / 2)-1} \rho_{i}^{2}
\end{aligned}
$$


and equating the expression for $p(\tilde{r})$ with the original expression given in (67) gives

$$
\begin{aligned}
q_{\circ} & =1 \\
q_{1} & =q_{\circ} \rho_{i}^{2}+c_{1} \\
q_{2} & =q_{1} \rho_{i}^{2}+c_{2} ; \cdots \\
q_{(d / 2)-1} & =q_{(d / 2)-2} \rho_{i}^{2}+c_{(d / 2)-1} .
\end{aligned}
$$

Hence, (70) can be rewritten as

$$
\begin{aligned}
& \frac{p(\tilde{r})}{\tilde{r}^{2}-\rho_{i}^{2}}=\tilde{r}^{d-2}+\left(\rho_{i}^{2}+c_{1}\right) \tilde{r}^{d-4}+\left(\rho_{i}^{4}+c_{1} \rho_{i}^{2}+c_{2}\right) \tilde{r}^{d-6} \\
& +\cdots+\left(\rho_{i}^{d-2}+c_{1} \rho_{i}^{d-4}+c_{2} \rho_{i}^{d-6}+\cdots\right. \\
& \left.+c_{(d / 2)-2} \rho_{i}^{2}+c_{(d / 2)-1}\right) \text {. }
\end{aligned}
$$

Equation (69) can now be written as

$$
\begin{aligned}
\frac{p^{\prime}(\tilde{r})}{2 \tilde{r}} \equiv & \sum_{i=1}^{d / 2} \frac{p(\tilde{r})}{\tilde{r}^{2}-\rho_{i}^{2}} \\
= & \frac{d}{2} \tilde{r}^{d-2}+\left(\sum_{i=1}^{d / 2} \rho_{i}^{2}+\frac{d}{2} c_{1}\right) \tilde{r}^{d-4} \\
& +\left(\sum_{i=1}^{d / 2} \rho^{4}+c_{1} \sum_{i=1}^{d / 2} \rho_{i}^{2}+\frac{d}{2} c_{2}\right) \tilde{r}^{d-6} \\
& +\cdots+\left(\sum_{i=1}^{d / 2} \rho_{i}^{d-2}+c_{1} \sum_{i=1}^{d / 2} \rho_{i}^{d-4}+\cdots\right. \\
& \left.+c_{(d / 2)-2} \sum_{i=1}^{d / 2} \rho_{i}^{2}+\frac{d}{2} c_{(d / 2)-1}\right)
\end{aligned}
$$

Now, by straightforward differentiation of (67) with respect to $\tilde{r},(69)$ can also be written as

$$
\begin{aligned}
\frac{p^{\prime}(\tilde{r})}{2 \tilde{r}}= & \frac{d}{2} \tilde{r}^{d-2}+\frac{c_{1}(d-2)}{2} \tilde{r}^{d-4}+\frac{c_{2}(d-4)}{2} \tilde{r}^{d-6} \\
& +\cdots+2 c_{(d / 2)-2} \tilde{r}^{2}+c_{(d / 2)-1}
\end{aligned}
$$

Comparing the coefficients of (74) and (75) gives rise to the following expressions for the coefficients of the SLP in terms of sums of the powers of the roots:

$$
\begin{aligned}
& c_{1}=-\xi_{2} ; \\
& c_{2}=-\frac{1}{2}\left(\xi_{4}+c_{1} \xi_{2}\right) ; \\
& c_{3}=-\frac{1}{3}\left(\xi_{6}+c_{1} \xi_{4}+c_{2} \xi_{2}\right) ; \cdots \\
& \quad \text { where } \xi_{n} \triangleq \sum_{i=1}^{d / 2} \rho_{i}^{n}=\frac{1}{2} \operatorname{sum}\left(\underline{\rho}^{n}\right) .
\end{aligned}
$$

In addition, in general, the $m$ th coefficient of the SLP can be written as

$$
c_{m}=-\frac{1}{m} \sum_{i=0}^{m-1} c_{i} \xi_{2(m-i)}
$$

Note that $\xi_{n}$ is the sum of the $n$th power of all the positive or negative roots (since $n$ is always even) of the SLP.

\section{APPENDIX B}

\section{DERIVATION OF SUM POWERS OF SENSOR LOCATIONS IN TERMS OF MANIFOLD CURVATURES}

The compact expression for the $i$ th manifold curvature $\kappa_{i}$ given by (21) can be expanded as

$$
\begin{aligned}
& \left(\kappa_{1} \kappa_{2} \cdots \kappa_{i-1} \kappa_{i}\right)^{2} \\
& =\left(b_{i, 1} \tilde{r}_{x 1}^{i+1}-b_{i, 2} \tilde{r}_{x 1}^{i-1}+\cdots\right. \\
& \left.\quad+(-1)^{\mathrm{fix}(i / 2)} b_{i, \mathrm{fix}(i / 2)+1} \tilde{r}_{x 1}^{i-2 \mathrm{fix}(i / 2)+1}\right)^{2}+\cdots \\
& \quad+\left(b_{i, 1} \tilde{r}_{x N}^{i+1}-b_{i, 2} \tilde{r}_{x N}^{i-1}+\cdots\right. \\
& \left.\quad+(-1)^{\mathrm{fix}(i / 2)} b_{i, \mathrm{fix}(i / 2)+1} \tilde{r}_{x N}^{i-2 \mathrm{fix}(i / 2)+1}\right)^{2} \cdot
\end{aligned}
$$

Note that (78) involves the sums of even powers of the normalized sensor locations up to and including the $2(i+1)$ th power. This implies that the sum of the $2(i+1)$ th power of normalized sensor locations $\operatorname{sum}\left(\underline{\tilde{r}}^{2(i+1)}\right)$ can be expressed in terms of the first $i$ manifold curvatures and the sums of lower even powers of the normalized sensor locations. Based on a similar reasoning, the sums of lower even powers of the normalized sensor locations must also be expressible in terms of the lower order curvatures, and hence, $\operatorname{sum}\left(\underline{\tilde{r}}^{2(i+1)}\right)$ can be explicitly written in terms of the first $i$ manifold curvatures only. The aim is to derive a general expression for $\operatorname{sum}\left(\underline{\tilde{r}}^{n}\right)$, where $n$ is even. The solution lies in the expression for the $((n / 2)-1)$ th curvature, which can be rearranged as

$$
\begin{aligned}
\left(\kappa_{1} \kappa_{2}\right. & \left.\cdots \kappa_{(n / 2)-1}\right)^{2} \\
= & \sum_{i=1}^{N}\left(b_{1} \tilde{r}_{x i}^{n / 2}-b_{2} \tilde{r}_{x i}^{(n / 2)-2}+\cdots+(-1)^{\mathrm{fix}(((n / 2)-1) / 2)}\right. \\
& \left.\cdot b_{\mathrm{fix}(((n / 2)-1) / 2)+1} \tilde{r}_{x i}^{(n / 2)-2 \mathrm{fix}(((n / 2)-1) / 2)}\right)^{2}
\end{aligned}
$$

where, for convenience

$$
b_{k} \triangleq b_{(n / 2)-1, k} .
$$

The last term of (79) can be simplified to

$$
b_{\mathrm{fix}(((n / 2)-1) / 2)+1}^{2} \tilde{r}_{x i}^{n-4 \mathrm{fix}(((n / 2)-1) / 2)}
$$

and further to

$$
\begin{cases}b_{\mathrm{fix}(((n / 2)-1) / 2)+1}^{2} \tilde{r}_{x i}^{4}, & \text { if } n \text { is divisible by } 4 \\ b_{\mathrm{fix}(((n / 2)-1) / 2)+1}^{2}{ }^{2}{ }_{x i}^{2}, & \text { otherwise. }\end{cases}
$$

Now, by expanding and rearranging (79), $\operatorname{sum}\left(\underline{\tilde{r}}^{n}\right)$ can be written as

$$
\begin{aligned}
\operatorname{sum}\left(\underline{\tilde{r}}^{n}\right)= & \left(\kappa_{1} \kappa_{2} \cdots \kappa_{(n / 2)-1}\right)^{2}+2 b_{1} b_{2} \operatorname{sum}\left(\underline{\tilde{r}}^{n-2}\right) \\
& -\left(2 b_{1} b_{3}+b_{2}^{2}\right) \operatorname{sum}\left(\underline{\underline{r}}^{n-4}\right)+2\left(b_{1} b_{4}+b_{2} b_{3}\right) \\
& \cdot \operatorname{sum}\left(\underline{\tilde{r}}^{n-6}\right)+\cdots-b_{\mathrm{fix}(((n / 2)-1) / 2)+1}^{2} \widetilde{r}_{x i}^{(4 \text { or } 2)} .
\end{aligned}
$$

To derive a general form of the above expression, consider two arbitrary terms, for instance, the $\ell$ th and $m$ th terms, from the bracketed expression in (79), given, respectively, by

$$
\begin{aligned}
& (-1)^{\ell-1} b_{\ell} \tilde{r}_{x i}^{(n / 2)-2(\ell-1)} \\
& (-1)^{m-1} b_{m} \tilde{r}_{x i}^{(n / 2)-2(m-1)} .
\end{aligned}
$$


These terms are actually squared and cross multiplied in (79), giving rise to the following expressions:

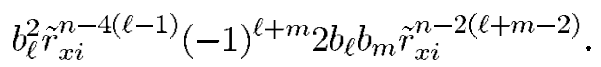

Based on the above result, the coefficient of a generic term $\left[\operatorname{sum}\left(\underline{\hat{r}}^{n-p}\right)\right]$ in $(83)$ can now be written as

$$
\begin{aligned}
\eta_{p}= \begin{cases}-\left(b_{(p / 4)+1}^{2}+2 \sum_{\ell, m} b_{\ell} b_{m}\right), & \text { if } p \text { is divisible by } 4 \\
2 \sum_{\ell, m} b_{\ell} b_{m}, & \text { otherwise }\end{cases} \\
\quad \text { where } m=\left(\frac{p}{2}+2\right)-\ell ; m>\ell ; \ell=1,2, \cdots .
\end{aligned}
$$

Using (86), the coefficients of $\operatorname{sum}\left(\underline{\tilde{r}}^{n-2}\right), \operatorname{sum}\left(\underline{\hat{r}}^{n-4}\right)$ and $\operatorname{sum}\left(\underline{\tilde{r}}^{n-6}\right)$ for instance, are, respectively, given by $\eta_{2}=2 b_{1} b_{2}$, $\eta_{4}=-\left(b_{2}^{2}+2 b_{1} b_{3}\right), \eta_{6}=2\left(b_{1} b_{4}+b_{2} b_{3}\right)$, as can be verified from (83). In summary, the sum of the $n$th power of the normalized sensor locations, where $n$ is even, can be written in terms of the first $((n / 2)-1)$ curvatures as follows

$$
\begin{aligned}
\operatorname{sum}\left(\underline{\tilde{r}}^{n}\right)= & \left(\kappa_{1} \kappa_{2} \cdots \kappa_{(n / 2)-1}\right)^{2}+2 b_{1} b_{2} \operatorname{sum}\left(\underline{\hat{r}}^{n-2}\right) \\
& -\left(2 b_{1} b_{3}+b_{2}^{2}\right) \operatorname{sum}\left(\underline{\underline{r}}^{n-4}\right)+\cdots \\
& +\eta_{p} \operatorname{sum}\left(\underline{\hat{r}}^{n-p}\right)+\cdots+b_{\text {fix }(((n / 2)-1) / 2)+1}^{2} \tilde{r}_{x i}^{q}
\end{aligned}
$$

where

$$
\begin{aligned}
& b_{k}=\sum_{m_{1}=1}^{(n / 2)-2 k+2} \sum_{m_{2}=m_{1}+2}^{(n / 2)-2 k+4} \cdots \sum_{m_{k-1}=m_{k-2}+2}^{(n / 2)-2} \\
& \text { - } \kappa_{m_{1}}^{2} \kappa_{m_{2}}^{2} \cdots \kappa_{m_{k-1}}^{2}, \quad k>2
\end{aligned}
$$

with

$$
\begin{aligned}
b_{1} & =1 ; \quad b_{2}=\sum_{i=1}^{(n / 2)-1} \kappa_{i}^{2} \\
q & = \begin{cases}4, & \text { if } n \text { is divisible by } 4 \\
2, & \text { otherwise }\end{cases} \\
\eta_{p} & =\text { given by }(86) .
\end{aligned}
$$

\section{REFERENCES}

[1] Y. T. Lo, "A mathematical theory of antenna arrays with randomly spaced elements," IEEE Trans. Antennas Propagat., vol. AP-12, pp. $257-268,1964$

[2] R. M. Leahy and B. D. Jeffs, "On the design of maximally sparse beamforming arrays," IEEE Trans. Antennas Propagat., vol. 39, pp. 1178-1187, Aug. 1991.

[3] G. L. Duckworth, S. W. Lang, and J. H. McClellan, "Array design for mem and mlm array processing," in Proc. IEEE Int. Conf. Acoust., Speech, Signal Process., vol. 1, Mar. 1981, pp. 145-148.

[4] X. Huang, J. P. Reilly, and M. Wong, "Optimal design of linear array of sensors," in Proc. IEEE Int. Conf. Acoust., Speech, Signal Process., May 1991, pp. 1405-1408.

[5] G. Nemhauser, M. I. Skolnik, and J. W. Sherman, "Dynamic programming applied to unequally spaced arrays," IEEE Trans. Antennas Propagat., vol. AP-12, pp. 35-43, 1964.

[6] R. L. Haupt, "Thinned arrays using genetic algorithms," IEEE Trans. Antennas Propagat., vol. 42, pp. 993-999, July 1994.
[7] V. Murino, "Simulated annealing approach for the design of unequally spaced arrays," in Proc. IEEE Int. Conf. Acoust., Speech, Signal Process., May 1995, pp. 3627-3630.

[8] C. S. Ruf, "Numerical annealing of low-redundancy linear arrays," IEEE Trans. Antennas Propagat., vol. 41, pp. 85-90, Jan. 1993.

[9] A. Alexiou, A. Manikas, and H. R. Karimi, "Comparison of the ultimate direction-finding capabilities of a number of planar array geometries," Proc. Inst. Elect. Eng., Radar, Sonar, Navig., vol. 144, no. 6, pp. 321-329, Dec. 1997.

[10] A. Manikas and C. Proukakis, "Modeling and estimation of ambiguities in linear arrays," IEEE Trans. Signal Processing, vol. 46, pp. 2166-2179, Aug. 1998.

[11] I. Dacos and A. Manikas, "The use of differential geometry in estimating the manifold parameters of a one-dimensional array of sensors," J. Franklin Inst., Eng. Appl. Math., vol. 332B, no. 3, pp. 307-332, 1995

[12] A. Manikas, H. R. Karimi, and I. Dacos, "Study of the detection and resolution capabilities of a one-dimensional array of sensors by using differential geometry," Proc. Inst. Elect. Eng., Radar, Sonar, Navig., vol. 141, no. 2, pp. 83-92, Apr. 1994.

[13] H. R. Karimi and A. Manikas, "The array manifold of a planar array and its effects on the accuracy of direction-finding systems," Proc. Inst. Elect. Eng., Radar, Sonar, Navig., vol. 143, no. 6, pp. 349-357, Dec. 1996.

[14] P. Stoica and A. Nehorai, "Music, maximum likelihood and Cramer-Rao bound," IEEE Trans. Acoust., Speech, Signal Processing, vol. 37, pp. 720-741, May 1989.

[15] W. S. Burnside and A. W. Panton, Theory of Equations. New York: Dover, 1960, vol. I/II

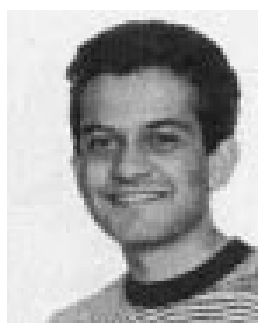

Naushad Dowlut (A'98) received the B.Eng.(Hons) degree in information systems engineering in 1992 and the Ph.D. degree in digital communications in 1997, both from Imperial College of Science, Technology and Medicine, University of London, London, U.K.

He held a postdoctoral fellowship at the Communications Research Laboratory, McMaster University, Hamilton, Ont., Canada, from 1998 to 1999. He is currently with Toronto Design Centre, Motorola Canada, Toronto, Ont., Canada. His research interests include array signal processing, wireless communications, and smart antennas.

Dr. Dowlut was the recipient of a Commonwealth Postgraduate Scholarship from 1993 to 1996 and an IEE Postgraduate Award in 1997.

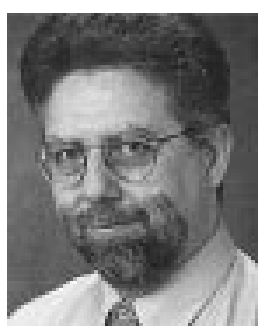

Athanassios Manikas (M'88) received the Ph.D and D.I.C. degrees from Imperial College of Science, Technology and Medicine, University of London, London, U.K., in 1988.

He was appointed Lecturer at Imperial College in 1988. He is now Reader in digital communications with the Department of Electrical and Electronic Engineering, Imperial College. He has published extensive sets of journal and conference papers relating to his research interests, which are in the general area of communication and signal processing, where he has developed a wide and deep interest in the topic of super-resolution array processing and the application of array theory to communication systems. $\mathrm{He}$ has been involved in array manifold investigations using differential geometry, super-resolution beamformers, direction finding, "near" and "far" field array processing, performance bounds in array systems, array uncertainties and ambiguities, array design, array signal abnormalities and quality factors, higher order signal subspace techniques, $H_{\infty}$-type robust aray processing, robust array receivers, and integrated wireless array communication networks based on code division multiple access, GSM-like, etc., platforms. Futhermore, he is the Organizer of the Advanced Communications and Signal Processing Laboratory, Imperial College, and, over the past ten years, has held a number of important research consultacies for the EU, industry, and government organizations. He is currently the Deputy Head of the Communications and Signal Processing Group at Imperial College.

Dr. Manikas is a corporate member of IEE and a Chartered Electrical Engineer. 